AJR Am J Roentgenol. 2016 July ; 207(1): 58-66. doi:10.2214/AJR.15.15995.

\title{
Emerging Applications of Abdominal 4D Flow MRI
}

\author{
Alejandro Roldán-Alzate ${ }^{1,2,3}$, Christopher J. Francois ${ }^{1}$, Oliver Wieben ${ }^{1,4}$, and Scott B. \\ Reeder ${ }^{1,2,3,4,5}$ \\ ${ }^{1}$ Department of Radiology, University of Wisconsin Madison, 1111 Highland Ave, 2494 WIMR2, \\ Madison, WI 53705 \\ 2Department of Mechanical Engineering, University of Wisconsin, Madison, WI \\ ${ }^{3}$ Department of Biomedical Engineering, University of Wisconsin, Madison, WI \\ ${ }^{4}$ Department of Medical Physics, University of Wisconsin, Madison, WI \\ ${ }^{5}$ Department of Emergency Medicine, University of Wisconsin, Madison, WI
}

\begin{abstract}
OBJECTIVE-Comprehensive assessment of abdominal hemodynamics is crucial for many clinical diagnoses but is challenged by a tremendous complexity of anatomy, normal physiology, and a wide variety of pathologic abnormalities. This article introduces 4D flow MRI as a powerful technique for noninvasive assessment of the hemodynamics of abdominal vascular territories.
\end{abstract}

CONCLUSION-Four-dimensional flow MRI provides clinicians with a more extensive and straightforward approach to evaluate disorders that affect blood flow in the abdomen. This review presents a series of clinical cases to illustrate the utility of 4D flow MRI in the comprehensive assessment of the abdominal circulation.

\section{Keywords}

4D flow MRI; abdominal hemodynamics; cirrhosis; meal challenge; renal artery stenosis

Comprehensive assessment of abdominal hemodynamics is crucial for many clinical diagnoses but is challenged by a tremendous complexity of anatomy, normal physiology, and a wide variety of pathologic abnormalities. Doppler ultrasound is the most commonly used diagnostic tool for noninvasive assessment of the abdominal circulation [1]. This technique can measure blood flow in large abdominal vessels by measuring velocities with high temporal resolution and approximating the cross-sectional area of the vessel, thereby estimating the average flow. Collateral pathways, which are associated with different cardiovascular pathologic abnormalities (e.g., portal hypertension, anatomic variations, and tumors), are highly variable and extend throughout the abdomen. Ultrasound is often limited for visualizing collateral (variceal) blood flow because of overlying intestinal gas and a limited acoustic window, which can lead to incomplete or inaccurate characterization of the abdominal hemodynamics. The role of ultrasound for the rapid determination of patency in

Address correspondence to A. Roldán-Alzate (roldan@wisc.edu).

A data supplement for this article can be viewed in the online version of the article at: www.ajronline.org. 
large vessels (e.g., main portal vein [PV]) cannot be questioned; however, its limitations for flow quantification and accurate depiction of variceal pathways are well known [2, 3].

Conventional flow-sensitive MRI using 2D slice selection, cardiac gating, and phasecontrast velocity encoding in one direction is an excellent quantitative alternative technique for measuring blood flow within the abdomen. This approach is frequently used for clinical applications that measure cardiac output and ejection fraction. It has also been validated for direct flow measurements in the azygos vein and main PV [4-6]. However, cardiac-gated 2D phase-contrast MRI requires operator expertise to select the vessel of interest and the use of double-oblique imaging planes that are challenging and time consuming to position and coordinate with patient breath-holding. Indeed, the acquisition of numerous $2 \mathrm{D}$ planes needed for comprehensive flow evaluation of the abdominal vasculature is not feasible in a clinical scenario. The need to represcribe 2D imaging planes on follow-up studies would also compromise the precision (repeatability) of 2D phase velocity MRI, limiting its utility as a quantitative biomarker of blood flow in the abdomen.

Time-resolved 3D phase-contrast MRI with three-directional flow encoding, also referred to as "4D flow MRI," has become a valuable research tool to investigate not only vascular anatomy but also blood flow and velocity in different vascular territories [7-15]. Fourdimensional flow MRI using cardiac-gated 3D spatial encoding throughout the cardiac cycle offers the unique combination of coregistered anatomic and hemodynamic visualization and quantification in a single examination. Velocity information acquired with 4D flow MRI can be used to derive various flow-related parameters, including pulse wave velocity, pressure gradients, and wall shear stress [16]. Several studies have been performed to validate 4D flow MRI by comparing it with 2D phase-contrast MRI [17], laser Doppler velocimetry [18], and ultrasound flow measurements in different vascular territories [7, 19].

Hemodynamic analysis of the portal venous system with 4D flow MRI using traditional Cartesian spatial encoding has shown promising results [15, 20,21]. The need to perform velocity encoding in three directions quadruples the scanning time over a non-velocityencoded acquisition and consequently limits the achievable spatial resolution and coverage within a reasonable scanning time. Often, the imaging volume is chosen to assess the flow only in large vessels to reduce scanning time, precluding the comprehensive assessment of large organs, such as the liver, and the evaluation of collateral flow pathways that span large anatomic regions. Furthermore, a single velocity-encoding setting limits the evaluation of flow to the portal and splanchnic venous vasculature. Alternatively, 3D radial undersampling strategies have shown the feasibility of both qualitative and quantitative flow assessment of the mesenteric hemodynamics with high spatial resolution and large volumetric coverage in reasonable scanning times, on the order of 10-15 minutes [22-25]. More recently, a method combining spiral sampling with compressed sensing reconstruction introduced a major acceleration to the 4D flow MRI acquisition in a single breath-hold, although with limited spatial and temporal resolution [26].

Four-dimensional flow MRI has moved from being a research-only sequence to providing relevant hemodynamic and anatomic information for the clinical diagnosis of different cardiovascular abnormalities in the abdomen. The ability of 4D flow MRI to not only 
visualize flow distributions in complex abdominal vascular beds but also to quantify hemodynamics makes it an ideal technique for comprehensive assessment of the abdominal circulation. In this way, 4D flow MRI is now providing clinicians with a more straightforward approach, in which they can quantify, a posteriori, blood flow at any location of the FOV and have information on the direction of the flow, which is ideal for the assessment of collateral pathways in the abdominal circulation and, in this sense, is superior to angiography. The purpose of this article is to present a series of clinical cases to illustrate the utility of 4D flow MRI in the assessment of the hemodynamics in the abdominal circulation.

\section{Emerging Abdominal Applications}

Over the past 2 decades, a number of groups have described the application of 4D flow MRI for the assessment of arterial and venous hemodynamics in various anatomic regions in the human body, including the chest, abdomen, and cranial vasculature [12]. In the following sections, we discuss representative clinical cases for the different vascular territories and clinically relevant challenges that are often present in the abdominal circulation. The intention of this article is to introduce 4D flow MRI as a powerful technique that can be used for noninvasive assessment of the hemodynamics of abdominal vascular territories.

MRI

For all cases presented in this article, 4D flow MRI was performed with a 5-point radially undersampled phase-contrast acquisition (i.e., phase contrast with vastly undersampled isotropic projection reconstruction) because of its increased velocity sensitivity performance $[27,28]$ and complete coverage of the upper abdomen [22-25]. Studies were conducted on a clinical 3-T scanner (Discovery MR750, GE Healthcare) with a 32-channel phased-array body coil (catalog number NC004000, NeoCoil). The 3D volume was centered over the celiac axis for the hepatic cases and in the renal arteries for the renal cases. Fourdimensional flow MRI parameters were as follows: imaging volume, $32 \times 32 \times 24 \mathrm{~cm}$ spherical; $1.25-\mathrm{mm}$ acquired isotropic spatial resolution; TR/TE, 6.4/2.2; free breathing; and scanning time, 12 minutes [29]. The acquisition was respiratory gated with a bellows signal and an adaptive acceptance window of 50\%. Retrospective ECG gating was accomplished with recorded $\mathrm{R}$ wave locations from vector gating performed during the offline reconstruction.

For many of the clinical and research studies at our institution, patients receive an IV injection of 0.03-0.05 mmol $/ \mathrm{kg}$ gadofosveset trisodium (Ablavar, Lantheus) or $0.1 \mathrm{mmol} / \mathrm{kg}$ gadobenate dimeglumine (Multihance, Bracco Diagnostics). Gadofosveset trisodium is an intravascular gadolinium-based contrast agent with weak protein binding, and gadobenate is well known to have protein association. In addition to increasing the relaxivity of these agents through reduced molecular tumbling, interaction with serum proteins also lengthens clearance from the blood, and these agents act as blood-pool agents for the duration of the 4D flow MRI acquisition. As a result, we are able to improve the image signal-to-noise ratio and velocity-to-noise ratio performance [30, 31]. It should be noted, however, that all 4D flow MRI methods, like 2D phase-contrast MRI, are inherently unenhanced MRI methods 
and do not require the use of gadolinium-based agents. When using gadolinium, the flip angle is increased to capitalize on the increased signal-to-noise ratio and velocity-to-noise ratio performance afforded by the $\mathrm{T} 1$ shortening associated with gadolinium.

\section{Four-Dimensional Flow MRI Visualization and Quantification}

During the retrospective offline reconstruction, the number of time frames is determined; that is, for analysis of portal venous flow, the cardiac cycle is divided into 14 time frames because pulsatility does not play a key role [23], and for arterial flow, it is divided into 20 time frames. Phase offsets for Maxwell terms and eddy currents are corrected automatically $[32,33]$. The eddy current correction is performed using second-order polynomial fitting of background tissue segmented on the basis of thresholding of an angiogram [33]. Velocityweighted angiograms are calculated from the final velocity and magnitude data for all time frames [34]. Manual placement of cut-planes in the vessel of interest is performed for subsequent flow quantification and visualization in EnSight (version 10.0, CEI). It is important to mention that other commercial software packages are currently being developed to visualize and quantify $4 \mathrm{D}$ flow MRI data.

\section{Liver}

Cirrhosis is the end-stage pathway of chronic liver disease leading to mortality of more than 35,000 people in the United States each year [1]. Portal hypertension is the most common and most lethal complication in patients with cirrhosis [1]. Dramatic alterations in blood flow occur in patients with cirrhosis during the development of portal hypertension. Initially, there is a progressive increase in vascular resistance at the sinusoidal level due to passive resistance caused by architectural changes related to fibrosis and active resistance related to vasoconstriction of vascular smooth muscle cells in the liver [35]. Portal hypertension can lead to portosystemic collateral pathways (varices), ascites, hepatorenal syndrome, portal venous thrombosis, and splenomegaly [36]. The presence of gastroesophageal varices is an important predictor of mortality and decompensation of cirrhosis [37]. A comprehensive diagnostic approach that assesses detailed hemodynamic and morphologic information with complete upper abdominal vascular coverage in a single examination would be highly desirable to further our understanding and serve as a noninvasive biomarker of disease progression and therapeutic monitoring.

Comprehensive noninvasive quantification of blood flow in the hepatic vasculature is challenging. The vascular anatomy of the liver is complex and highly variable, and its morphologic features and flow may be severely altered in the presence of portal hypertension. The ability of radial $4 \mathrm{D}$ flow MRI to cover large imaging volumes makes it a suitable technique for assessing the complex hemodynamics of the liver both in physiologic and pathologic conditions.

Case 1: collateral flow and portal shunt fraction-Four-dimensional flow MRI is very useful in assessing the hemodynamic changes that occur in patients with cirrhosis (Figs. 1 and 2). Hepatofugal (away from the liver) blood flow in the large paraumbilical collateral as well as in the coronary vein draining from the PV can occur when portal venous pressures and resistance to portal venous flow increase. The main advantage of $4 \mathrm{D}$ flow 
MRI in complex clinical applications is its ability to provide comprehensive qualitative (anatomy and flow patterns) and quantitative (flow measurements) assessment of the abdomen. In case 1, the blood flow in the PV, superior mesenteric vein (SMV), splenic vein (SV), and collateral vessels was quantified, and the shunt fraction in the liver (percentage of total portal blood flow bypassing the liver through collateral pathways) was calculated as $Q_{C o l} /\left(Q_{S M V}+Q_{S V}\right)$, where $Q_{C o l}$ is the total collateral flow (coronary vein plus paraumbilical collateral), $Q_{S M V}$ is the blood flow in the SMV, and $Q_{S V}$ is the blood flow in the SV. In this patient, $90 \%$ of the total blood flow to the liver through the PV ( $Q_{S M V}+$ $\left.Q_{S V}\right)$, bypassed the liver through the collateral vessels. This suggests elevated resistance in the liver, which translates into severe portal hypertension according to the Ohm law for fluid flow $(\Delta P=Q \times R)$, where $\Delta P$ is the pressure gradient between the portal venous system and the systemic venous system, $R$ is the resistance to blood flow in the portal circulation, and $Q$ is the blood flow.

Case 2: hepatofugal flow-Varices, which are anatomic alterations in the portal venous system, appear in response to pathologically increased vascular resistance in patients with portal hypertension. In agreement with the anatomic changes in the mesenteric and portal vasculature, velocity color-coded streamlines and arrowheads reveal the presence of hepatofugal flow in the SV in Figure 2 (see also Video S1, which can be viewed in the AJR electronic supplement to this article available at www.ajronline.org). Blood flow quantification in the SMV $\left(Q_{S M V}=0.5 \mathrm{~L} / \mathrm{min}\right), \mathrm{SV}\left(Q_{S V}=-0.36 \mathrm{~L} / \mathrm{min}\right)$, and PV $\left(Q_{P V}=\right.$ $0.14 \mathrm{~L} / \mathrm{min}$ ) revealed a shunt fraction of $72 \%$. Note the negative flow in the SV indicating that the flow occurred in the hepatofugal direction. Velocity streamlines show that the blood flow in the SV drains into the splenorenal shunt, bypassing the liver to avoid the high resistance created by the cirrhosis.

Case 3: meal challenge-Ingestion of food is physiologically followed by vasodilatation and increased mesenteric blood flow, a phenomenon known as postprandial hyperemia [38]. Meal challenges are standard clinical procedures applied in imaging modalities such as ultrasound and MRI to induce physiologic hyperemia of the gut. A recent study combined 4D flow MRI and a meal challenge to further deepen the understanding of hepatic physiology and to present a biomarker for a given pathologic abnormality - specifically, portal hypertension [25]. Increased flow can be easily visualized in the portal and splanchnic circulation before and after a meal in healthy volunteers (Fig. 3). This study concluded that portal venous regulation, as a response to increasing mesenteric venous flow after a meal challenge, may be impaired in patients with cirrhosis. At our institution, the meal challenge protocol includes an initial 4D flow MRI study after a period of fasting (prechallenge study) of at least 5 hours (no food, liquid, or chewing gum). After the first study, patients ingest 574 $\mathrm{mL}$ of EnSure Plus (Abbott Laboratories; 700 calories, 28\% from fat and 57\% from carbohydrates) [39-41]. A second 4D flow MRI study (postchallenge scan) is then repeated 20 minutes after the meal ingestion.

To illustrate the effect of a meal on portal venous flow in patients with portal hypertension, case 3 is presented in Figure 4 (see also Videos S2A and S2B, which can be viewed in the $A J R$ electronic supplement to this article available at www.ajronline.org). A patient with 
alcoholic cirrhosis and cystic fibrosis-related liver disease underwent a meal challenge 4D

flow MRI test to evaluate the hemodynamic response of mesenteric and portal circulation to food ingestion. In this patient, the portal venous flow was hepatopetal (i.e., blood flows toward the liver) before and after the meal challenge; however, the coronary vein blood flow was hepatofugal both before and after the meal, draining from the SV into the esophageal varices, which is a clinical sign of portal hypertension. Interestingly, before the meal, blood flow in the SV changed direction and became hepatofugal, draining into the coronary vein as shown by the velocity streamlines in Figure 4 .

\section{Case 4: living donor liver transplantation surgical planning and follow-up-}

Liver transplantation is a highly successful therapy for patients with end-stage liver failure. In the past 2 decades, the growing disparity between the number of liver transplant candidates and the supply of deceased donor organs has motivated the implementation of living donor liver transplantation [42]. More recently, improvements in this procedure have rendered results comparable to those from deceased donors. However, a low platelet count in donor blood samples could suggest an increase in portal venous pressure. This is expected when recognizing that a resection of hepatic tissue translates into an increase in vascular resistance, which is directly proportional to pressure as described by the Ohm law for fluid flow. The ability to quantify hemodynamic changes in the portal and mesenteric circulation noninvasively shows that 4D flow MRI may be a suitable tool for both surgical planning of living donor liver transplantation and improved understanding of the hemodynamic changes that occur in the liver remnant of the donor.

As part of the clinical protocol for surgical planning of living donor liver transplantation, 4D flow MRI is used to visualize and quantify the inflow and outflow to the liver. Figure 5 shows the flow and anatomy visualization in an example case of right-lobe resection. Threedimensional volume-rendered images from a complex difference dataset of 4D flow MRI acquisitions obtained before and after surgery are shown in Figures 5A and 5B. Velocity streamlines representing the flow distribution in the portal venous system are shown in Figures 5C and 5D for pre- and postsurgery studies, respectively. The quantitative analysis revealed an increase in the portal venous flow $(0.81 \mathrm{vs} 1.3 \mathrm{~L} / \mathrm{min}) 2$ weeks after surgery. The blood flow in the remaining left PV increased sevenfold $(0.061 \mathrm{vs} 0.53 \mathrm{~L} / \mathrm{min})$. These changes agree with previously reported data [43] showing that one of the acute consequences of a partial hepatectomy is the increase in blood flow to the liver, which helps in the regeneration of the remaining tissue.

Case 5: transjugular intrahepatic portosystemic shunt-When medical therapies are no longer effective, portal hypertension can be treated using a transjugular intrahepatic portosystemic shunt (TIPS). This shunt diverts blood flow from the portal system directly into the systemic circulation, reducing portal pressure and thereby helping to resolve ascites and reduce the risk of variceal hemorrhage [44, 45]. Unfortunately, excessive shunting of portal blood into the systemic circulation is associated with the risk of developing post-TIPS hepatic encephalopathy [46]. Another major complication is the recurrence of portal hypertension secondary to the development of in-stent stenosis, despite improved patency with the advent of covered stents [47, 48]. Two-dimensional Doppler ultrasound plays a 
central role in monitoring patients before and after TIPS placement; however, its limitations make it a suboptimal method to fully characterize the blood flow, not only in the portal system before TIPS but also in the shunt after TIPS to evaluate patency. Four-dimensional flow MRI has been used successfully for monitoring of hepatic blood flow in patients with portal hypertension [20, 24].

A patient with idiopathic liver cirrhosis and refractory ascites after TIPS implantation was studied. A 4D flow MRI study was performed before and 2 weeks after the TIPS implantation. Figure 6 (see also Videos S3A and S3B, which can be viewed in the AJR electronic supplement to this article available at www.ajronline.org) shows the velocity distribution in the portal system by means of color-coded streamlines, revealing slow flow in the main PV and right PV as well as high flow in the left PV. In this case, the flow pattern was caused by arterioportal-venous shunting draining from a peripheral branch of the left hepatic artery into a branch of the left PV. This arterioportal-venous shunt induced the highest measured portosystemic gradient of $28 \mathrm{~mm} \mathrm{Hg}$. TIPS further increased the flow in the LPV because of the shunt, resulting in the fastest observed flow in the TIPS with only a slight reduction of the portosystemic gradient to $18 \mathrm{~mm} \mathrm{Hg}$. As a result, this patient had refractory ascites even after implantation of TIPS.

\section{Kidneys}

Renal artery stenosis is a recognized cause of hypertension and progressive renal insufficiency, occurring in up to $45 \%$ of patients with peripheral vascular disease [49]. Prompt assessment of the hemodynamic significance of renal artery lesions is clinically important for treatment planning and monitoring for patients with vascular disease as well as for kidney transplant donors and recipients. A lesion that narrows the luminal diameter by $75 \%$ or more is usually hemodynamically significant. In cases of a mild stenosis $(<50 \%)$, no intervention is typically required. However, the hemodynamic significance of a stenosis measured as $50-75 \%$ cannot be reliably derived from vessel diameter measurements alone; hence, intraarterial pressure measurements under x-ray angiography are often obtained. MRI of the renal vasculature is challenging because of the relatively small vessel diameter, especially of segmental branches and complex motion of the renal vessels throughout the cardiac and respiratory cycles.

Contrast-enhanced (CE) MR angiography (MRA) is commonly used for renal artery stenosis assessment. However, patients with compromised kidney function, including kidney transplant donors and recipients, should not receive gadolinium-based contrast agents because of the risk for nephrogenic systemic fibrosis [50]. Radial 4D flow MRI without the use of a contrast agent has been successfully applied to this vascular territory [8, 51]. Freebreathing acquisitions of 10 minutes provide angiograms of both renal arteries and veins with good correlation of diameter measures across various vessel sizes [8] as an alternative to CE-MRA (Fig. 7). Furthermore, radial 4D flow MRI showed superior visualization of segmental renal arteries because of decreased kidney-vessel contrast in CE-MRA from parenchymal enhancement. In addition, the functional information can be used for the noninvasive assessment of hemodynamic significance of renal artery stenosis. As validated 
in an animal study, transstenotic pressure gradients derived from the velocity fields correlated well with measurements obtained invasively with pressure wires [51].

Recently, 4D flow MRI was used in a kidney transplant recipient for evaluation of the patency of the right renal artery. Figure 8 (case 6) (see also Video S4, which can be viewed in the $A J R$ electronic supplement to this article available at www.ajronline.org) shows the qualitative and quantitative information about the blood flow through the left renal artery. In the proximal section of the artery, a decrease in velocity signal shows the location of the stent; however, excellent agreement in the conservation of mass analysis shows that 4D flow MRI is a feasible technique to quantify blood flow downstream of a stent, not only in renal arteries but also in any other vascular territory.

\section{Mesenteric Ischemia}

A variety of diseases can lead to chronic mesenteric ischemia, which is characterized by abdominal pain that appears about 15-60 minutes after a meal as a result of inadequate blood flow. The most common cause is atherosclerosis, in which a narrowing of the celiac and superior mesenteric arteries limits blood flow to the intestines. However, other causes exist, such as median arcuate ligament syndrome, superior mesenteric syndrome, clots, and venous thrombus. Evaluation of mesenteric ischemia is complicated by the rich network of mesenteric collateral pathways available from the superior and inferior mesenteric arteries, which, in some patients, may compensate for decreases in flow through the celiac axis.

We routinely use 4D flow MRI in our clinical practice to improve diagnosis by providing simultaneous anatomic depiction and functional assessment of the hemodynamics in the entire upper abdomen before and after a meal challenge. To illustrate the range of conditions observed, two patients will be discussed in more detail.

For case 7, a patient with celiac trunk stenosis and poststenotic aneurysm underwent 4D flow MRI before and after a meal challenge. Figure 9 shows the volume-rendered phase contrast angiogram and streamline visualization with peak systolic flow before and after a meal challenge. In this patient, flow in the gastroduodenal artery was reversed and increased by $28 \%$ after the meal, while wall shear stress within the aneurysm increased 4.5 -fold after the meal. The second patient (case 8) had collateral circulation through the arc of Riolan when scanned for mesenteric ischemia (Fig. 10). In this patient, the mesenteric flow did not change drastically in response to the meal; however, the collateral blood flow through the arc of Riolan increased fourfold.

\section{Discussion}

Four-dimensional flow MRI is an evolving technology that has recently gained increased importance because of its potential to provide a comprehensive evaluation of vascular hemodynamics with full volumetric coverage, not only in the abdominal circulation but also in other vascular territories [12]. Although pulse sequences for 4D flow MRI continue to be developed, its accuracy and test-retest reliability have been and are constantly seen in various phantom and in vivo studies [17, 24, 52-54]. Even though our institution is actively using 4D flow MRI clinically for different complex hemodynamic pathologic abnormalities, 
the 4D flow MRI techniques used on the clinical cases described in this article are not commonly available on routine clinical MRI systems. Most research groups active in the area of 4D flow MRI rely on MRI sequences that are not commercially available. Increased efforts by the MRI research community and the MRI system vendors are needed to afford a more widespread availability of optimized 4D flow MRI techniques.

In summary, 4D flow MRI, an emerging tool for the comprehensive evaluation of cardiovascular hemodynamics with full volumetric coverage, is a continuously developing field of research. Current implementations permit its integration into clinical protocols on the premises that scan times need to be further reduced and subsequent data analysis is successfully integrated into the clinical workflow. Overall, the increasing amount of available studies and applications of 4D flow MRI to comprehensive analysis of the abdominal circulation reveal its potential for an improved understanding of the hemodynamics involved in different diseases involving the abdominal circulation.

\section{Supplementary Material}

Refer to Web version on PubMed Central for supplementary material.

\section{Acknowledgments}

Supported by grants R01 DK096169, R01 DK096169, and K24 DK 102595 from the National Institutes of Health (NIH) National Institute of Diabetes and Digestive and Kidney Diseases, grant R01 R01HL072260 from the NIH National Heart, Lung, and Blood Institute, the University of Wisconsin Research and Development Fund, GE Healthcare, and Bracco Diagnostics.

\section{References}

1. de Vries PJ, van Hattum J, Hoekstra JB, de Hooge P. Duplex Doppler measurements of portal venous flow in normal subjects: inter- and intraobserver veriability. J Hepatol. 1991; 13:358-363. [PubMed: 1808227]

2. Thabut D, Moreau R, Lebrec D. Noninvasive assessment of portal hypertension in patients with cirrhosis. Hepatology. 2011; 53:683-694. [PubMed: 21274889]

3. Vizzutti F, Arena U, Rega L, et al. Performance of Doppler ultrasound in the prediction of severe portal hypertension in hepatitis C virus-related chronic liver disease. Liver Int. 2007; 27:1379-1388. [PubMed: 18036101]

4. Gouya H, Mallet V, Scatton O, et al. Magnetic resonance imaging (MRI) flow, a new non-invasive method for the evaluation of systemic, splanchnic, and azygos blood flows in patients with portal hypertension (abstr). Hepatology. 2008; 48(suppl 5):1049A.

5. Burkart DJ, Johnson CD, Ehman RL, Weaver AL, Ilstrup DM. Evaluation of portal venous hypertension with cine phase-contrast MR flow measurements: high association of hyperdynamic portal flow with variceal hemorrhage. Radiology. 1993; 188:643-648. [PubMed: 8351326]

6. Wu MT, Pan HB, Chen C, et al. Azygos blood flow in cirrhosis: measurement with MR imaging and correlation with variceal hemorrhage. Radiology. 1996; 198:457-462. [PubMed: 8596849]

7. Chang W, Landgraf B, Johnson KM, et al. Velocity measurements in the middle cerebral arteries of healthy volunteers using 3D radial phase-contrast HYPRFlow: comparison with transcranial Doppler sonography and 2D phase-contrast MR imaging. AJNR. 2011; 32:54-59. [PubMed: 20947642]

8. François CJ, Lum DP, Johnson KM, et al. Renal arteries: isotropic, high-spatial-resolution, unenhanced MR angiography with three-dimensional radial phase contrast. Radiology. 2011; 258:254-260. [PubMed: 20980449] 
9. Francois CJ, Srinivasan S, Schiebler ML, et al. 4D cardiovascular magnetic resonance velocity mapping of alterations of right heart flow patterns and main pulmonary artery hemodynamics in tetralogy of Fallot. J Cardiovasc Magn Reson. 2012; 14:16. [PubMed: 22313680]

10. Nett, EJ.; Johnson, KM.; Francois, CJ.; Lunn, D.; Wieben, O. Accelerated dual velocity-encoded phase contrast VIPR. In: Sodickson, DK., editor. Proceedings of International Society for Magnetic Resonance in Medicine (ISMRM) 2010 annual meeting. Montreal, QC, Canada: ISMRM; 2010.

11. Wieben, O.; Block, WF.; Barger, V.; Grist, TM.; Mistretta, C. XIV Annual International Workshop on MR Angiography. Essen, Germany: Society for Magnetic Resonance Angiography; 2002. 3D Cardiac cine imaging with vastly undersampled isotropic projection imaging (VIPR).

12. Markl M, Frydrychowicz A, Kozerke S, Hope M, Wieben O. 4D flow MRI. J Magn Reson Imaging. 2012; 36:1015-1036. [PubMed: 23090914]

13. Barker AJ, Roldan-Alzate A, Entezari P, et al. Four-dimensional flow assessment of pulmonary artery flow and wall shear stress in adult pulmonary arterial hypertension: results from two institutions. Magn Reson Med. 2015; 73:1904-1913. [PubMed: 24974951]

14. Stankovic Z, Blanke P, Markl M. Usefulness of 4D MRI flow imaging to control TIPS function. Am J Gastroenterol. 2012; 107:327-328. [PubMed: 22306955]

15. Stankovic Z, Frydrychowicz A, Csatari Z, et al. MR-based visualization and quantification of three-dimensional flow characteristics in the portal venous system. J Magn Reson Imaging. 2010; 32:466-475. [PubMed: 20677279]

16. Wentland AL, Grist TM, Wieben O. Review of MRI-based measurements of pulse wave velocity: a biomarker of arterial stiffness. Cardiovasc Diagn Ther. 2014; 4:193-206. [PubMed: 24834415]

17. Wentland AL, Grist TM, Wieben O. Repeatability and internal consistency of abdominal 2D and 4D phase contrast MR flow measurements. Acad Radiol. 2013; 20:699-704. [PubMed: 23510798]

18. Hollnagel DI, Summers PE, Kollias SS, Poulikakos D. Laser Doppler velocimetry (LDV) and 3D phase-contrast magnetic resonance angiography (PC-MRA) velocity measurements: validation in an anatomically accurate cerebral artery aneurysm model with steady flow. J Magn Reson Imaging. 2007; 26:1493-1505. [PubMed: 17968887]

19. Eriksson J, Carlhäll CJ, Dyverfeldt P, Engvall J, Bolger AF, Ebbers T. Semi-automatic quantification of 4D left ventricular blood flow. J Cardiovasc Magn Reson. 2010; 12:9. [PubMed: 20152026]

20. Stankovic Z, Csatari Z, Deibert P, et al. Normal and altered three-dimensional portal venous hemodynamics in patients with liver cirrhosis. Radiology. 2012; 262:862-873. [PubMed: 22357888]

21. Stankovic Z, Fink J, Collins JD, et al. K-t GRAPPA-accelerated 4D flow MRI of liver hemodynamics: influence of different acceleration factors on qualitative and quantitative assessment of blood flow. MAGMA. 2015; 28:149-159. [PubMed: 25099493]

22. Frydrychowicz A, Landgraf BR, Niespodzany E, et al. Four-dimensional velocity mapping of the hepatic and splanchnic vasculature with radial sampling at 3 Tesla: a feasibility study in portal hypertension. J Magn Reson Imaging. 2011; 34:577-584. [PubMed: 21751287]

23. Landgraf BR, Johnson KM, Roldán-Alzate A, Francois CJ, Wieben O, Reeder SB. Effect of temporal resolution on 4D flow MRI in the portal circulation. J Magn Reson Imaging. 2014; 39:819-826. [PubMed: 24395121]

24. Roldán-Alzate A, Frydrychowicz A, Niespodzany E, et al. In vivo validation of 4D flow MRI for assessing the hemodynamics of portal hypertension. J Magn Reson Imaging. 2013; 37:1100-1108. [PubMed: 23148034]

25. Roldán-Alzate A, Frydrychowicz A, Said A, et al. Impaired regulation of portal venous flow in response to a meal challenge as quantified by 4D flow MRI. J Magn Reson Imaging. 2015; 42:1009-1017. [PubMed: 25772828]

26. Dyvorne H, Knight-Greenfield A, Jajamovich G, et al. Abdominal 4D flow MR imaging in a breath hold: combination of spiral sampling and dynamic compressed sensing for highly accelerated acquisition. Radiology. 2015; 275:245-254. [PubMed: 25325326]

27. Gu T, Korosec FR, Block WF, et al. PC VIPR: a high-speed 3D phase-contrast method for flow quantification and high-resolution angiography. AJNR. 2005; 26:743-749. [PubMed: 15814915] 
28. Johnson KM, Markl M. Improved SNR in phase contrast velocimetry with five-point balanced flow encoding. Magn Reson Med. 2010; 63:349-355. [PubMed: 20099326]

29. Roldán-Alzate, A.; Frydrychowicz, A.; Niespodzany, EJ.; Landgraf, BR.; Wieben, O. 4D MR velocity mapping using PC VIPR to quantify blood flow in portal hypertension (abstr). In: Sodickson, DK., editor. Proceedings of International Society for Magnetic Resonance in Medicine (ISMRM) 2010 annual meeting. Montreal, QC Canada: ISMRM; 2010.

30. Bock J, Frydrychowicz A, Stalder AF, et al. 4D phase contrast MRI at 3 T: effect of standard and blood-pool contrast agents on SNR, PC-MRA, and blood flow visualization. Magn Reson Med. 2010; 63:330-338. [PubMed: 20024953]

31. Landgraf, B.; Frydrychowicz, A.; Johnson, K., et al. 4D PC MR of the portal venous system: benefits of using a blood pool contrast agent. (abstr). In: Sodickson, DK., editor. Proceedings of International Society for Magnetic Resonance in Medicine (ISMRM) 2010 annual meeting. Montreal, QC, Canada: ISMRM; 2010.

32. Bernstein MA, Xiaohong JZ, Zhou J, et al. Concomitant gradient terms in phase contrast MR: analysis and correction. Magn Reson Med. 1998; 39:300-308. [PubMed: 9469714]

33. Walker PG, Cranney GB, Scheidegger MB, Waseleski G, Pohost GM, Yoganathan AP. Semiautomated method for noise reduction and background phase error correction in MR phase velocity data. J Magn Reson Imaging. 1993; 3:521-530. [PubMed: 8324312]

34. Johnson KM, Lum DP, Turski PA, Block WF, Mistretta CA, Wieben O. Improved 3D phase contrast MRI with off-resonance corrected dual echo VIPR. Magn Reson Med. 2008; 60:1329_ 1336. [PubMed: 19025882]

35. Bhathal PS, Grossman HJ. Reduction of the increased portal vascular resistance of the isolated perfused cirrhotic rat liver by vasodilators. J Hepatol. 1985; 1:325-337. [PubMed: 4056346]

36. Bari K, Garcia-Tsao G. Treatment of portal hypertension. World J Gastroenterol. 2012; 18:11661175. [PubMed: 22468079]

37. D'Amico G, Garcia-Tsao G, Pagliaro L. Natural history and prognostic indicators of survival in cirrhosis: a systematic review of 118 studies. J Hepatol. 2006; 44:217-231. [PubMed: 16298014]

38. Chou CC. Splanchnic and overall cardiovascular hemodynamics during eating and digestion. Fed Proc. 1983; 42:1658-1661. [PubMed: 6832382]

39. Li KC, Hopkins KL, Dalman RL, Song CK. Simultaneous measurement of flow in the superior mesenteric vein and artery with cine phase-contrast MR imaging: value in diagnosis of chronic mesenteric ischemia—work in progress. Radiology. 1995; 194:327-330. [PubMed: 7824706]

40. Lycklama à Nijeholt GJ, Burggraaf K, Wasser MN, et al. Variability of splanchnic blood flow measurements using MR velocity mapping under fasting and post-prandial conditions: comparison with echo-Doppler. J Hepatol. 1997; 26:298-304. [PubMed: 9059950]

41. Sidery MB, Macdonald IA, Blackshaw PE. Superior mesenteric artery blood flow and gastric emptying in humans and the differential effects of high fat and high carbohydrate meals. Gut. 1994; 35:186-190. [PubMed: 8307468]

42. Brown RS Jr, Russo MW, Lai M, et al. A survey of liver transplantation from living adult donors in the United States. N Engl J Med. 2003; 348:818-825. [PubMed: 12606737]

43. Yag S, Iida T, Taniguchi K, et al. Effect of portal venous pressure on regeneration and graft damage after living donor liver transplantation. Liver Transl. 2005; 11:68-75.

44. Garcia-Tsao G, Bosch J. Management of varices and variceal hemorrhage in cirrhosis. N Engl J Med. 2010; 362:823-832. [Erratum in N Engl J Med 2001; 364:490]. [PubMed: 20200386]

45. Boyer TD, Haskal ZJ. American Association for the Study of Liver Diseases. The role of transjugular intrahepatic portosystemic shunt (tips) in the management of portal hypertension: update 2009. Hepatology. 2010; 51:306. [PubMed: 19902484]

46. Riggio O, Nardelli S, Moscucci F, Pasquale C, Ridola L, Merli M. Hepatic encephalopathy after transjugular intrahepatic portosystemic shunt. Clin Liver Dis. 2012; 16:133-146. [PubMed: 22321469]

47. Perarnau JM, Le Gouge A, Nicolas C, et al. Covered vs. uncovered stents for transjugular intrahepatic portosystemic shunt: a randomized controlled trial. J Hepatol. 2014; 60:962-968. [PubMed: 24480619] 
48. Chen S, Li X, Wei B, et al. Recurrent variceal bleeding and shunt patency: prospective randomized controlled trial of transjugular intrahepatic portosystemic shunt alone or combined with coronary vein embolization. Radiology. 2013; 268:900-906. [PubMed: 23657891]

49. Missouris CG, Buckenham T, Cappuccio FP, MacGregor GA. Renal artery stenosis: a common and important problem in patients with peripheral vascular disease. Am J Med. 1994; 96:10-14. [PubMed: 8304356]

50. Agarwal R, Brunelli SM, Williams K, Mitchell MD, Feldman HI, Umscheid CA. Gadoliniumbased contrast agents and nephrogenic systemic fibrosis: a systematic review and meta-analysis. Nephrol Dial Transplant. 2009; 24:856-863. [PubMed: 18952698]

51. Bley TA, Johnson KM, François CJ, et al. Noninvasive assessment of transstenotic pressure gradients in porcine renal artery stenoses by using vastly undersampled phase-contrast MR angiography. Radiology. 2011; 261:266-273. [PubMed: 21813739]

52. Wentland AL, Wieben O, Korosec FR, Haughton VM. Accuracy and reproducibility of phasecontrast MR imaging measurements for CSF flow. AJNR. 2010; 31:1331-1336. [PubMed: 20203113]

53. Markl M, Wallis W, Harloff A. Reproducibility of flow and wall shear stress analysis using flowsensitive four-dimensional MRI. J Magn Reson Imaging. 2011; 33:988-994. [PubMed: 21448968]

54. van Ooij P, Powell AL, Potters WV, Carr JC, Markl M, Barker AA. Reproducibility and interobserver variability of systolic blood flow velocity and 3D wall shear stress derived from 4D flow MRI in the healthy aorta. J Magn Reson Imaging. 2016; 43:236-248. [PubMed: 26140480] 


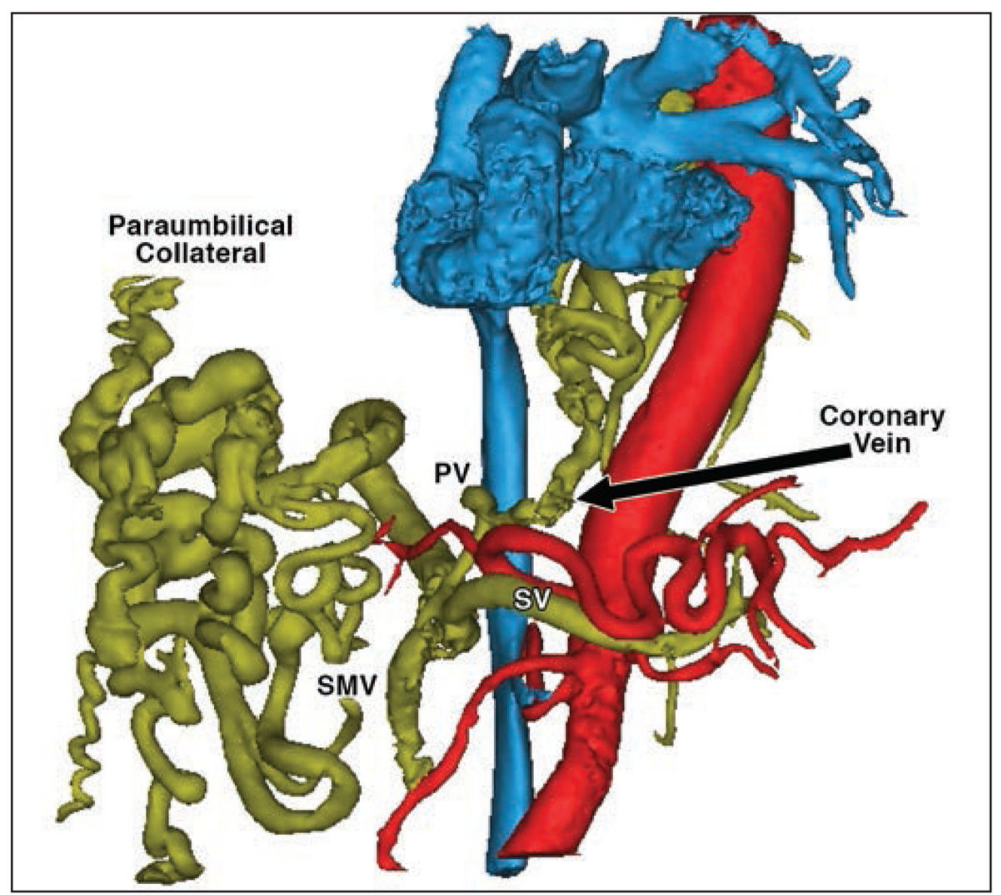

Fig. 1.

Segmentation of 4D flow MRI angiogram in left anterior oblique view shows arterial (red), venous (blue), and portal (yellow) circulations. Note collateral vessels in portal circulations. $\mathrm{PV}=$ portal vein, $\mathrm{SV}=$ splenic vein, $\mathrm{SMV}=$ superior mesenteric vein. (Illustration by Roldán-Alzate A) 


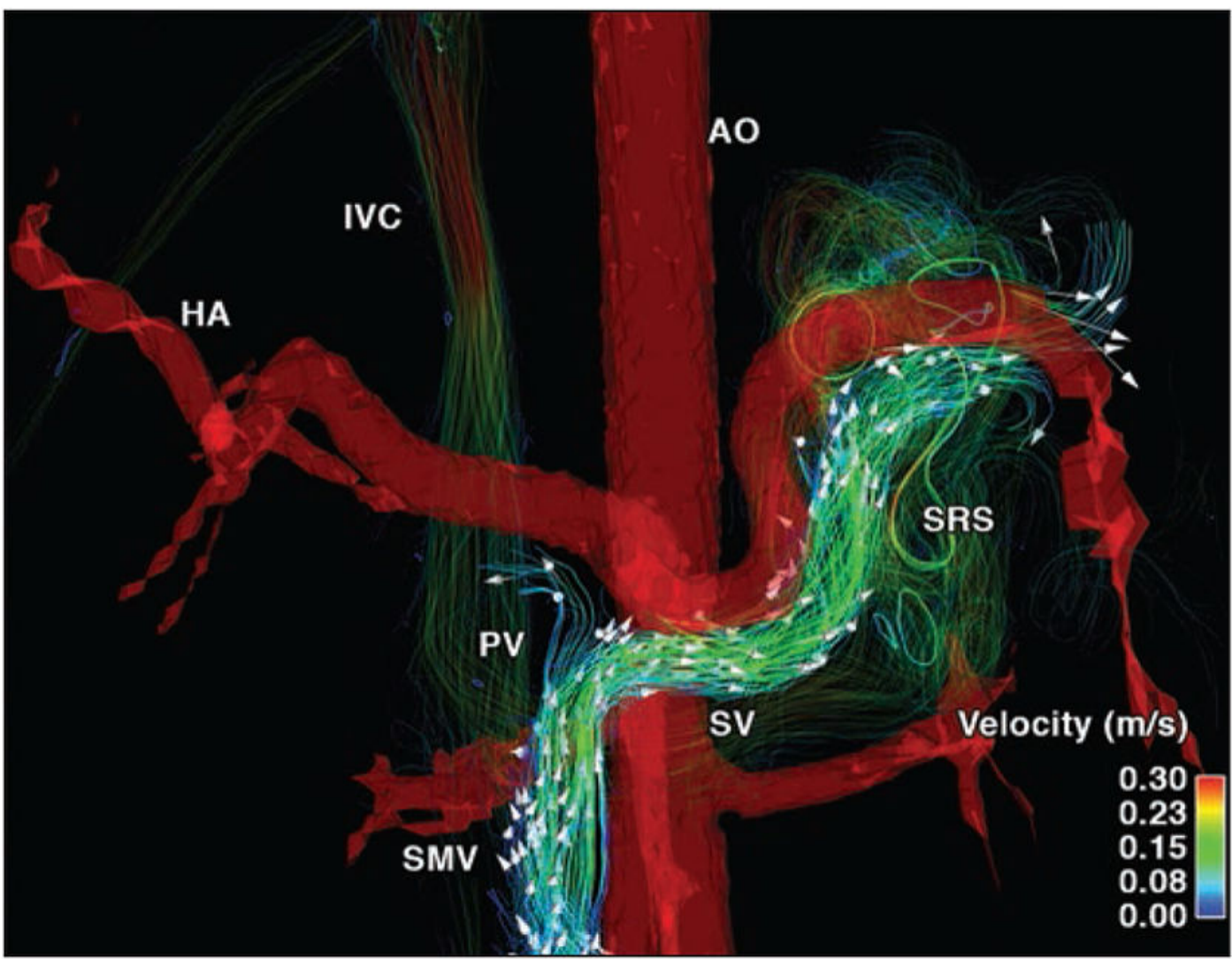

Fig. 2.

26-year-old woman $(67 \mathrm{~kg})$ with hepatic cirrhosis secondary to biliary atresia who underwent repair for biliary atresia as infant (Kasai procedure) (case 1). Segmentation of phase-contrast MR angiogram reveals presence of portosystemic shunts by means of splenorenal shunt (SRS). Hepatofugal flow in splenic vein (SV) can be observed (arrows). $\mathrm{AO}=$ abdominal aorta, $\mathrm{HA}=$ hepatic artery, $\mathrm{IVC}=$ inferior vena cava, $\mathrm{PV}=$ portal vein, SMV = superior mesenteric vein 


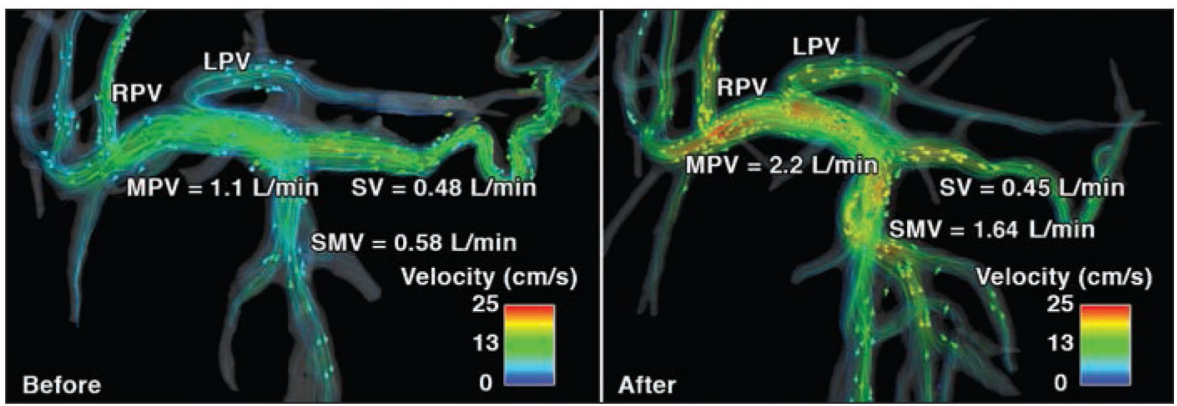

Fig. 3.

32-year-old man (79.5 kg) with no history of liver disease (case 2). Velocity distribution shown by velocity color-coded streamlines on 4D flow MR images obtained before (left) and after (right) meal challenge reveals blood flow increase in superior mesenteric vein (SMV), left portal vein (LPV), right portal vein (RPV), and main portal vein (MPV) in response to meal challenge. Arrowheads show direction of blood flow. Reduction in splenic vein (SV) flow can also be observed. 


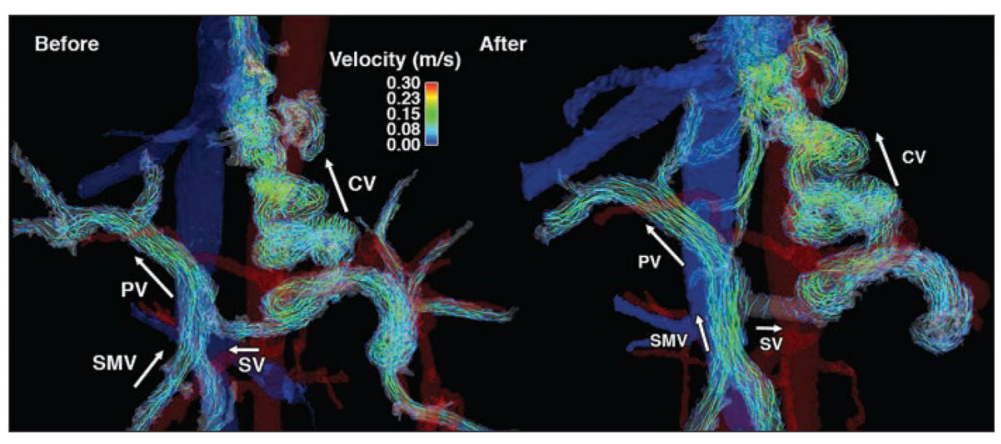

Fig. 4.

34 -year-old man $(61.2 \mathrm{~kg})$ with alcoholic cirrhosis and cystic fibrosis-related liver disease (case 3). Hepatopetal flow in portal vein (PV) was seen before (left) and after (right) meal challenge; however, hepatofugal blood flow was also seen both before (left) and after (right), meal through coronary vein $(\mathrm{CV})$. Arrows show blood flow direction. As response to meal challenge (right) slow splenic blood flow becomes hepatofugal and drains into CV. SMV = superior mesenteric vein, $\mathrm{SV}=$ splenic vein. 


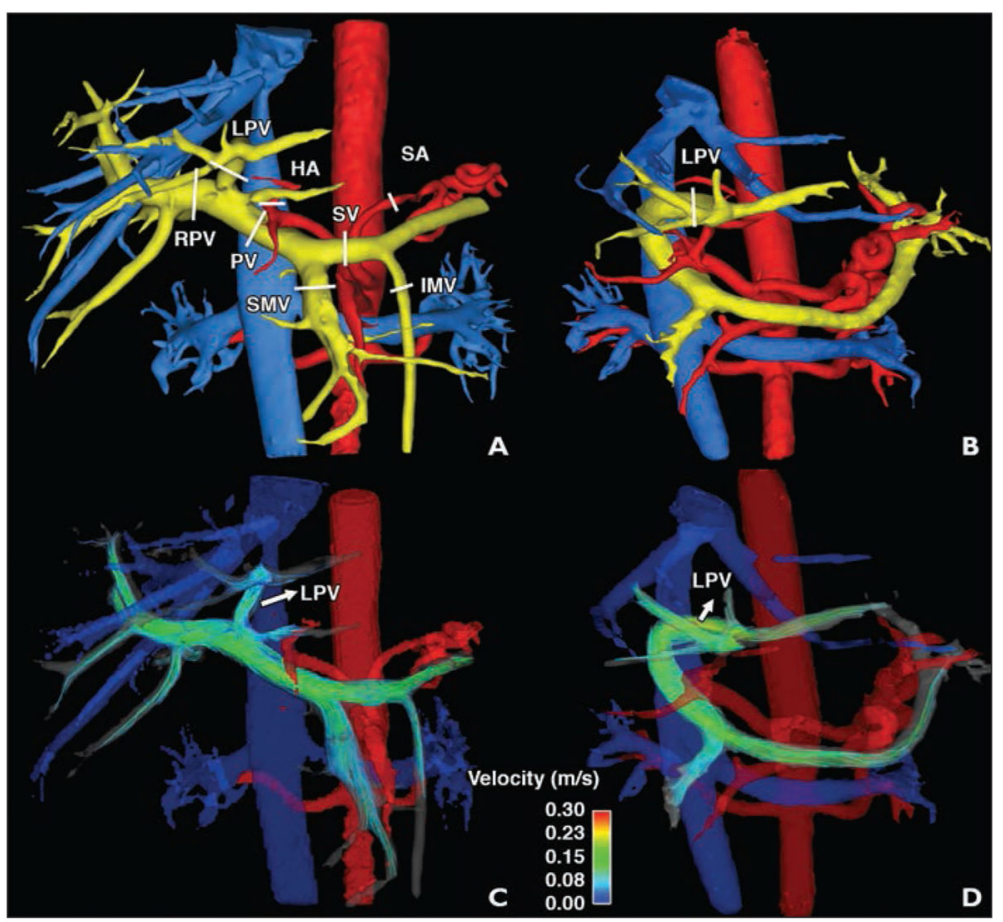

Fig. 5.

33-year-old woman (case 4). Images show flow and anatomy visualization in right lobe. A-D, Three-dimensional volume-rendered images were created from complex difference dataset of phase-contrast vastly undersampled isotropic projection imaging acquisition before (A and $\mathbf{C}$ ) and after ( $\mathbf{B}$ and $\mathbf{D})$ surgery. White lines (A and $\mathbf{B}$ ) show locations of measurement planes. Color-coded streamlines show velocity distribution (arrows, $\mathbf{C}$ and $\mathbf{D}$ ) in portal venous system. $\mathrm{LPV}=$ left portal vein, $\mathrm{RPV}=$ right portal vein, $\mathrm{PV}=$ portal vein, $\mathrm{SMV}=$ superior mesenteric vein, $\mathrm{SV}=$ splenic vein, $\mathrm{HA}=$ hepatic artery, $\mathrm{IMV}=$ inferior mesenteric vein, $\mathrm{SA}=$ splenic artery. 


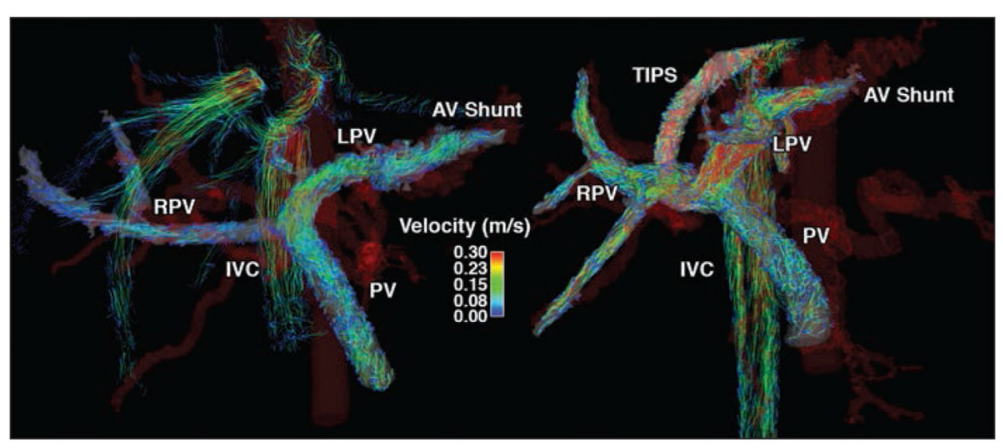

Fig. 6.

46-year-old man with idiopathic liver cirrhosis and refractory ascites before (left) and after (right) transjugular intrahepatic portosystemic shunt (TIPS) procedure (case 5). Velocity distribution in portal system shown by velocity color-coded streamlines reveals slow flow in main portal vein (PV) and right PV (RPV), and high flow in left PV (LPV). This flow pattern was caused by arterioportal-venous (AV) shunting draining from peripheral branch of left hepatic artery into branch of LPV. TIPS further increased flow in LPV due to shunt, which resulted in refractory ascites even after TIPS. IVC $=$ inferior vena cava. 


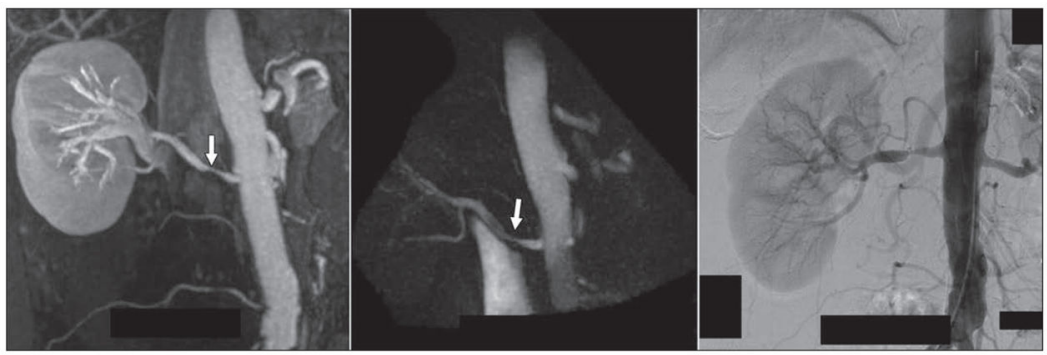

Fig. 7.

61-year-old woman with renal artery stenosis (arrows). Contrast-enhanced MR angiography (left), unenhanced 4D flow MRI angiography (middle), and digital subtraction angiography (right) images are shown for comparison. 


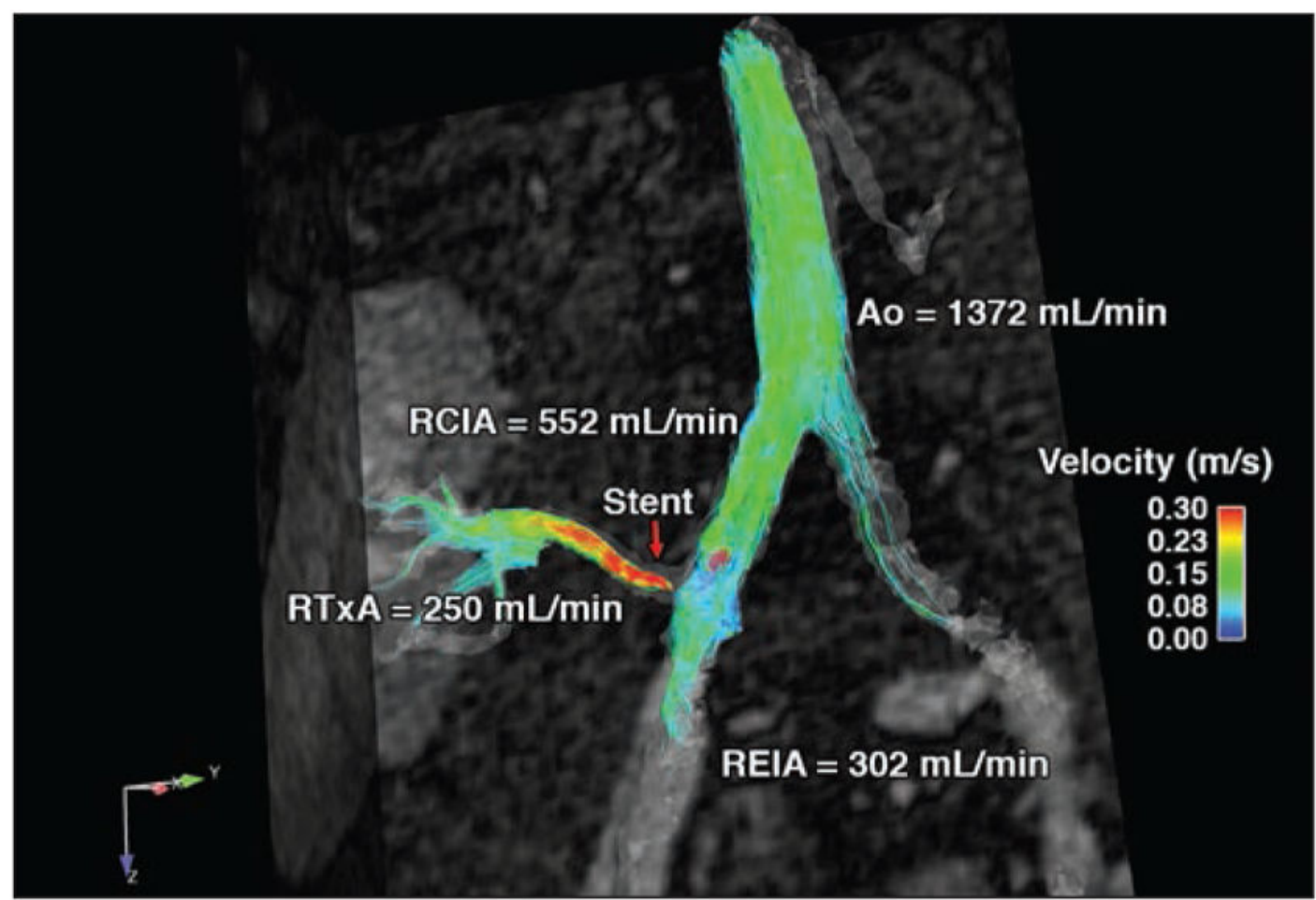

Fig. 8.

51-year-old man who underwent evaluation of renal artery flow after kidney transplant (case 6). Red arrow shows location of stent. Ao = abdominal aorta, RCIA = right common iliac artery, $\mathrm{RTxA}=$ right transplant artery, REIA= right external iliac artery. 

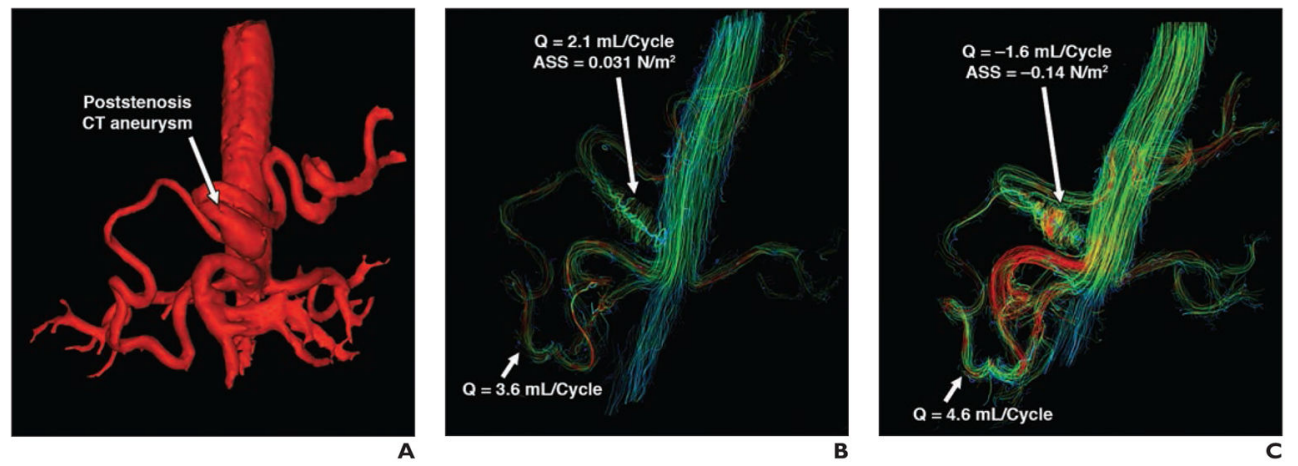

Fig. 9.

65-year-old man with celiac trunk stenosis and poststenotic aneurysm (case 7).

A-C, Volume-rendered phase-contrast angiogram (A) and streamline visualizations (left anterior oblique view) with peak systolic flow before (B) and after (C) meal challenge are shown. ASS = aneurysm shear stress, $Q=$ flow. 


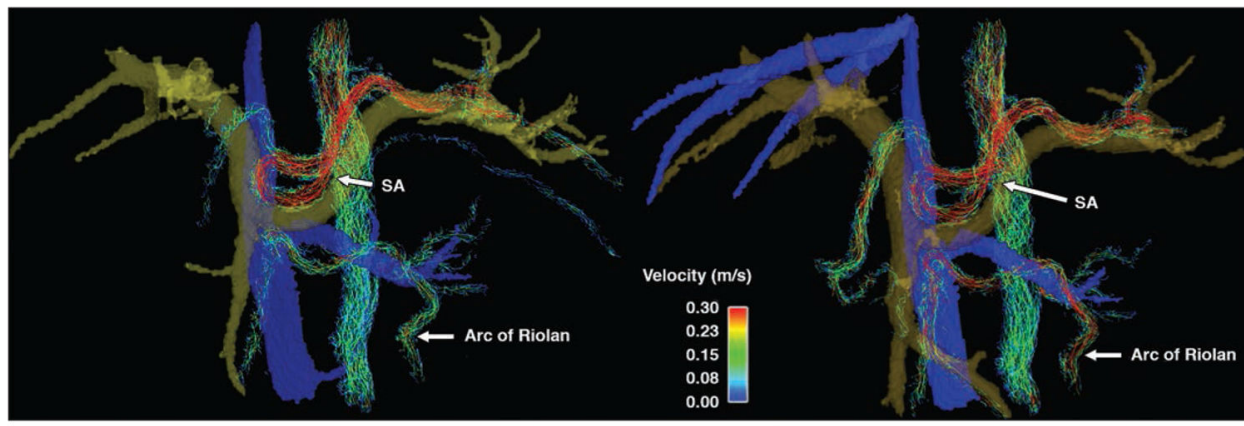

Fig. 10.

46-year-old man with irregular blood flow due to aortic repair (case 8). MR angiograms were obtained before (left) and after (right) meal challenge. Note collateral circulation through arc of Riolan, which dramatically increased in response to meal challenge. SA = splenic artery. 